\title{
Artigos
}

$\mathscr{C}$

\section{ACCOGLIERE FAMIGLIE CON BAMBINI DISABILI IN CONTESTI MIGRATORI. SCENARI INCLUSIVI A BOLOGNA}

\author{
Welcoming families with disabled children in migratory contexts. \\ Perspectives of integration in Bologna
}

Emanuela Bini*

\begin{abstract}
Riassunto. II numero crescente di famiglie migranti con minori disabili invita a riflettere sul tema dell'accoglienza e delle politiche sociali. Come evidenziato dalla ricerca "Alunni con disabilità, figli di mig ranti" condotta a Bologna, risulta necessario che i servizi alla persona e le istituzioni scolastiche - Enti che collaborano nella presa in carico del minore disabile - rinnovino le pratiche di accoglienza e di accompagnamento delle famiglie e dei minori disabili per rispondere in maniera adeguata ai bisogni emergenti. Considerato il numero esiguo di studi sul tema e la mancanza di linee guida ministeriali per definire interventi incentrati sulla "doppia diversità", le criticità e le buone prassi individuate dalla ricerca assumono un valore paradigmatico e forniscono utili indicazioni estendibili anche ad altri contesti.
\end{abstract}

Parole chiave: disabilità, migrazione, città, Istituzioni, integrazione.

Abstract. The increasing number of migrant families with disabled children invites to think about welcoming practices and social policies. As the research "Alunni con disabilità, figli di migranti" carried out in Bologna emphasizes, it is necessary that health services and school system renew welcoming practices to answer the rising needs of migrant families with disabled children. Difficulties and good inclusive practices underlined in the research assume a paradigmatic value and provide useful directions even for other places, considering that there is not a huge number of studies about disability in migratory contexts and that there are no Ministerial guidelines to define proper actions based on "double diversity".

Keywords: disability, migration, urban space, Institutions, integration.

* Laureata in Scienze della Formazione Primaria presso I'Università degli Studi di Bologna. Bologna, Italia. 


\section{Introduzione}

In Italia si è registrata nell'ultimo decennio una rapida ed intensa crescita quantitativa del fenomeno migratorio contraddistinto da una sempre maggiore stanzialità, come dimostrato dalla crescita della componente familiare e dall'aumento del numero di alunni figli di migranti, aspetti che hanno trasformato profondamente il paesaggio urbano, sempre più plurale e multiculturale. I flussi migratori infatti tendono a concentrarsi in alcune aree urbane del nostro Paese in particolare nelle principali città del Centro Nord, capaci di offrire maggiori opportunità occupazionali e una rete di servizi percepiti come più efficienti (trasporti pubblici, servizi socio-sanitari qualificati...).

Secondo il Ventitreesimo Rapporto sulle migrazioni curato dalla Fondazione ISMU (Iniziative e Studi sulla Multietnicità), nel 2017 Bologna risulta essere la città con la più elevata percentuale di cittadini stranieri per numero di residenti e per numero di alunni. I dati evidenziano che sotto le Due Torri il fenomeno migratorio sta assumendo sempre più un carattere strutturale, destinato a modificare gli scenari delle politiche sociali. Emerge inoltre la necessità di rispondere ai bisogni emergenti di una "nuova" fetta di popolazione: si tratta di nuclei familiari migranti con minori disabili iscritti nelle scuole bolognesi.

Secondo la ricerca "Alunni con disabilità, figli di migranti" condotta proprio a Bologna - uno dei primi studi sul tema della disabilità in contesti migratori per rispondere ai bisogni emergenti risulta necessario che le Istituzioni preposte alla presa in carico dei minori disabili - i servizi socio-sanitari e la scuola conoscano le condizioni di vita delle famiglie e adottino strategie adeguate per migliorare le pratiche di accoglienza ed implementare interventi a tutela del diritto alla salute e all'istruzione.

Ho deciso di approfondire il ruolo delle Istituzioni nella fase di accoglienza e di accompagnamento del minore disabile e della sua famiglia circoscrivendo il campo di indagine all'area metropolitana di Bologna per due motivi principali. In primo luogo, come accennato sopra, nel 2017 Bologna detiene due primati statistici che la collocano in cima alla lista delle città più multiculturali del nostro Paese. Il capoluogo emiliano è dunque una realtà urbana chiamata, ormai da diversi anni, ad interfacciarsi con richieste avanzate da un'utenza sempre più multiculturale, dinamica che sollecita un ripensamento dell'organizzazione dei servizi alla persona e delle politiche sociali. In secondo luogo, è stata condotta proprio a Bologna la ricerca "Alunni con disabilità, figli di migranti. Approcci culturali, questioni educative, prospettive inclusive" riguardante l'integrazione degli alunni disabili migranti. Si tratta di uno studio che ha indagato il ruolo dei servizi alla persona e delle istituzioni scolastiche nel percorso di integrazione, individuando criticità e buone prassi; di fronte all'odierna lacuna normativa a riguardo, la ricerca assume un valore paradigmatico e fornisce utili indicazioni generalizzabili anche ad altri contesti. 
Nel primo paragrafo illustro dati e tendenze del fenomeno migratorio a Bologna. Nei paragrafi successivi mi concentro sui dati emersi dalla ricerca "Alunni con disabilità, figli di migranti. Approcci culturali, questioni educative, prospettive inclusive", esiti che integrati con i recenti studi nel campo dell'antropologia, dell'etnografia e dell'approccio transculturale consentono di analizzare la situazione delle famiglie, le principali difficoltà, il ruolo delle Istituzioni e le buone prassi a sostegno dell'accoglienza e dell'inclusione.

\section{L'esperienza migratoria a Bologna: dati e tendenze}

II report pubblicato nel mese di Ottobre 2017 dall' "Osservatorio Regionale sul fenomeno migratorio" evidenzia alcuni aspetti interessanti. In primo luogo, l'Emilia-Romagna si conferma la regione con il maggior numero di stranieri residenti e con la più alta incidenza di cittadini provenienti da Paesi stranieri rispetto al totale della popolazione residente: con I'11,9\% I'EmiliaRomagna supera la media nazionale $(8,3 \%)$ e si piazza davanti alla Lombardia $(11,4 \%)$. In secondo luogo, Bologna e provincia registrano un boom di richieste di residenza; nel 2017 risultavano registrati 118.013 migranti (ben 770 in più rispetto al 2016), con un'incidenza del 15,4\%, la più alta della regione EmiliaRomagna. La comunità con più residenti è quella dei romeni (circa 89.000 residenti), pari al 16,7\% delle presenze straniere - comunità più numerosa anche a livello italiano. Al secondo posto c'è la comunità proveniente dal Marocco $(11,6 \%)$, al terzo posto gli albanesi $(11,0 \%)$, seguiti da ucraini $(6,1 \%)$, cinesi e moldavi (entrambi al 5,5\%) ${ }^{1}$.

Nella maggior parte dei casi, le motivazioni che spingono i migranti a raggiungere I'Italia e a stabilirsi soprattutto nelle regioni del Centro Nord, come l'Emilia-Romagna, sono di natura economica, riconducibili alla ricerca di un lavoro e alla speranza di migliorare le proprie condizioni socio-economiche. In molti casi emergono motivazioni più urgenti dal punto di vista umanitario: la fuga da un Paese in guerra o da realtà dove sono negati i basilari diritti umani (rifugiati e richiedenti asilo); la prospettiva di ricevere un'adeguata assistenza in caso di parenti malati o disabili².

Rispetto a qualche anno fa, i dati rilevano che in Emilia-Romagna il fenomeno dell'immigrazione sta assumendo un carattere sempre più strutturale

1 Osservatorio Regionale sul fenomeno migratorio. Rapporto Cittadini stranieri in Emilia Romagna. Residenti e dinamiche demografiche, p. 9-10.

2 Spesso le due motivazioni umanitarie possono coesistere; in questi casi la normativa prevede la possibilità di attivare dei corridoi umanitari per permettere ai richiedenti asilo in condizione di vulnerabilità (disabilità, gravi malattie) di raggiungere l'Europa con un regolare visto per motivi umanitari. Si tratta di un intervento volto a razionalizzare i flussi migratori e a sottrarre i richiedenti asilo al dramma dei viaggi clandestini per mare o per terra. In Italia i primi corridoi umanitari sono stati attivati nel biennio 2015-2016 attraverso un protocollo d'intesa tra il Ministero dell'Interno e la Comunità di Sant'Egidio (CODINI, Ennio. Gli aspetti normativi, p. 106-107). 
e meno temporaneo. Un primo aspetto che conferma questa tendenza è rappresentato dall'aumento del numero di stranieri che hanno acquisito la cittadinanza italiana ${ }^{3}$. Si tratta di un cambiamento in parte riconducibile ad un trend generale riscontrabile anche nelle altre regioni. Se infatti fino a qualche anno fa il nostro Paese rappresentava per i migranti provenienti dall'Africa e dal Medio Oriente un Paese di passaggio per raggiungere i Paesi del Nord Europa al fine di unirsi a parenti e accedere ad un mercato del lavoro più appetibile, oggi I'Italia rappresenta sempre più il contesto dove stabilirsi definitivamente considerate anche le politiche migratorie restrittive adottate da alcuni Stati europei negli ultimi mesi. Il carattere stanziale della migrazione trova ulteriore conferma in un secondo aspetto significativo: I'aumento del numero di alunni iscritti a scuola, dato che evidenzia l'intenzionalità della maggior parte delle famiglie migranti di stabilirsi definitivamente nel contesto di approdo.

Secondo il Ventitreesimo Rapporto sulle migrazioni 2017 curato dalla Fondazione ISMU e presentato a Milano lo scorso 5 Dicembre, Bologna è la città con le classi più multietniche d'Italia. Il capoluogo emiliano supera metropoli come Milano, Roma, Torino e Genova per presenza percentuale di bambini stranieri nelle scuole. In base ai dati forniti dal Ministero della Pubblica Istruzione, Bologna non solo risulta tra le prime dieci province per presenza di immigrati nelle scuole di ogni ordine e grado, ma è la prima per la concentrazione di stranieri che rappresentano il $15,4 \%$ degli iscritti. Una percentuale che supera il $14,7 \%$ di Milano, il $12,3 \%$ di Torino, il $12,1 \%$ di Genova e il $10 \%$ di Roma. Il Rapporto 2017 della Fondazione ISMU segnala inoltre che Bologna presenta la percentuale più alta - pari al $14 \%$ - di scuole che superano il tetto del $30 \%$ di alunni stranieri, tetto fissato dal Ministero dell'Istruzione in base al quale ogni anno l'Ufficio Scolastico Regionale consente agli Istituti delle deroghe per formare classi che ospitano un numero maggiore di alunni non italiani rispetto al tetto stabilito dal Ministero. Come nel resto d'Italia, anche a Bologna la percentuale maggiore di alunni stranieri si concentra nella scuola dell'infanzia ${ }^{4}$.

Le statistiche relative gli ultimi anni rilevano inoltre l'aumento del numero di alunni migranti con disabilità nelle scuole italiane. Secondo gli ultimi dati disponibili sulla disabilità in contesti migratori (anno scolastico 2014/15), gli studenti migranti disabili sono 28.117 (di cui 8.921 femmine) con un aumento rispetto al precedente anno pari a 1.491 unità (l'incidenza degli alunni stranieri sul totale degli studenti disabili è del $12 \%$ ), con valori al di sopra della media nella scuola dell'infanzia $(15,2 \%)$, nella scuola primaria $(13,8 \%)$ e nella scuola secondaria di primo grado $(12,6 \%)$. Si tratta di un fenomeno vario per età, origini

3 Osservatorio Regionale sul fenomeno migratorio, op. cit., p. 16.

4 CORNEO, Daniela. Alunni stranieri tra i banchi: Bologna supera Milano e Roma. II rapporto ISMU: qui il maggior numero di classi dove si sfora il tetto del 30\%. Corriere di Bologna on-line. 09.12.2017. 
culturali, tipo di deficit e incidenza territoriale. L'Emilia-Romagna si conferma tra le prime regioni per numerosità di presenze, dopo Lombardia e Veneto ${ }^{5}$.

Per una famiglia migrante con un minore disabile, il modello di integrazione degli studenti con disabilità nelle scuole comuni e la possibilità di accedere ad un'adeguata assistenza socio-sanitaria - condizioni non sempre garantite nei Paesi di origine - costituiscono valide motivazioni per stabilirsi in Italia sebbene siano spesso molte le difficoltà da affrontare nella quotidianità, come evidenziato dalla ricerca "Alunni con disabilità, figli di migranti" di seguito presentata ${ }^{6}$.

\section{La ricerca "Alunni con disabilità, figli di migranti. Approcci culturali, questioni educative, prospettive inclusive"}

Condotta sul territorio bolognese con la supervisione scientifica e il coordinamento della Prof.ssa Roberta Caldin, docente di Pedagogia Speciale presso l'Università di Bologna, la ricerca si è svolta dal 2008 al 2010. L'indagine si presenta come una ricerca-azione finalizzata ad indagare le condizioni delle famiglie migranti con minori disabili e il ruolo degli Enti preposti alla presa in carico dei bambini con disabilità nel percorso di integrazione.

Come previsto dalla Legge 104/1992 (Legge quadro per l'assistenza, I'integrazione sociale e i diritti delle persone handicappate), "la scuola e i servizi socio-sanitari sono le istituzioni chiamate a collaborare per sostenere lo sviluppo del bambino disabile attraverso interventi congiunti di natura riabilitativa ed educativa, volti a promuovere l'acquisizione delle competenze necessarie per la socializzazione, gli apprendimenti e le autonomie" ${ }^{\prime 7}$. Ai fini della ricerca, di notevole importanza è stato l'impegno dei ricercatori e degli esperti coinvolti per esplorare un terreno ancora poco conosciuto, per riflettere sui dati e sulle strategie da adottare per garantire la qualità degli interventi nella presa in carico di un soggetto migrante con disabilità.

Caratterizzata da un forte taglio interdisciplinare, la ricerca ha visto il coinvolgimento e la collaborazione tra diversi Enti: il Comune di Bologna, la Facoltà di Scienze della Formazione Primaria dell'Università di Bologna, I'Ufficio Scolastico Regionale, I'ASL di Bologna, I'Istituto dei Ciechi "Cavazza" e la Fondazione "Gualandi" di Bologna ${ }^{8}$. Il gruppo di ricerca ha privilegiato I'analisi di alcuni temi: le condizioni delle famiglie nel Paese di approdo (Argiropoulos), i servizi sanitari e territoriali (Leonardi), gli approcci educativi e didattici (Caldin, Dainese, Gori).

5 FONDAZIONE ISMU, MIUR. Rapporto nazionale sugli alunni con cittadinanza non italiana. La scuola multiculturale nei contesti locali, p. 37-40.

6 CALDIN, Roberta, ARGIROPOULOS, Dimitrios, DAINESE, Roberto. Genitori migranti e figli con disabilità. Le rappresentazioni dei professionisti e le percezioni delle famiglie, p. 17-18.

7 BINI, Emanuela. Famiglie migranti con minori disabili. Prospettive inclusive nel sistema sociosanitario e scolastico italiano, p. 209.

8 CALDIN, Roberta. Alunni con disabilità figli di migranti. Intrecci, contrasti, prospettive, p. 28. 
Per conoscere le condizioni delle famiglie, le modalità di accoglienza e gli approcci didattici, la ricerca ha analizzato 304 questionari (docenti della scuola dell'infanzia, primaria e secondaria di primo grado), focus group e interviste (36 insegnanti, 7 docenti di sostegno, 4 dirigenti scolastici, 33 genitori, 6 operatori sanitari).

La ricerca è stata uno dei primi studi che a livello nazionale hanno analizzato il tema della disabilità in contesti migratori ${ }^{9}$. Ad oggi l'indagine "Alunni con disabilità, figli di migranti" costituisce il principale quadro teorico di riferimento per conoscere ed approfondire le condizioni delle famiglie e il ruolo dei servizi socio-sanitari ed educativi nell'ambito dell'accoglienza e dell'integrazione. Sul tema della "doppia diversità" non esiste infatti una riflessione consolidata a livello normativo sebbene il fenomeno sia in aumento. Esistono documenti monotematici: Circolari Ministeriali e linee guida per l'accoglienza di alunni stranieri e Circolari Ministeriali e linee guida per alunni disabili; non esistono protocolli o documenti che si concentrano sulla "doppia diversità". Di fronte alla lacuna normativa sul tema, le indicazioni fornite dalla ricerca bolognese costituiscono utili riferimenti teorici e metodologici che - integrati con i recenti studi nel campo dell'antropologia e dell'etnografia - concorrono a delineare scenari inclusivi e orientamenti operativi.

L'attenzione alla famiglia e al percorso migratorio, uno degli aspetti trattati dall'indagine, risulta funzionale sia per descrivere il contesto in chiave sociologica sia per costruire un'impostazione metodologica sistemica. La famiglia è infatti il contesto primario del bambino, I'interlocutore privilegiato nella costruzione del progetto di vita e parte integrante del lavoro di rete con la scuola e i servizi socio-sanitari; i professionisti che prendono in carico la disabilità di un minore devono tener conto delle condizioni in cui vivono le famiglie e della dimensione culturale per orientare interventi adeguati.

\section{Genitori migranti con bambini disabili. Scenari di integrazione tra criticità e buone prassi}

Le storie delle famiglie raccolte dai ricercatori che hanno partecipato all'indagine "Alunni con disabilità, figli di migranti" rappresentano uno spaccato significativo di un mondo ancora poco indagato che i servizi socio-sanitari e la scuola dovrebbero conoscere per migliorare la relazione con le famiglie ed implementare interventi mirati. Secondo la ricerca, il rischio di esclusione sociale e le difficoltà di natura linguistica ed interpretativa sulle diverse rappresentazioni

\footnotetext{
9 In Italia esiste un numero esiguo di studi che hanno indagato la disabilità e la migrazione nel loro intersecarsi. Per un approfondimento della ricerca qui presentata, si rimanda al volume e alle riviste dedicati all'indagine: CALDIN, Roberta (a cura di). Alunni con disabilità, figli di migranti. Approcci culturali, questioni educative, prospettive inclusive. Riviste: Educazione Interculturale. Culture, esperienze, progetti, v. 9, n. 1, 2011; Ricerche di Pedagogia e Didattica, v. 5, n. 1, 2010".
} 
culturali dei concetti di disabilità e di cura rappresentano le due principali aree di criticità che possono compromettere un sereno rapporto con le famiglie.

Di seguito, analizzo le problematiche individuate nel contesto "domestico", nelle fasi di accoglienza e di accompagnamento. Di fronte alle difficoltà rilevate, illustro alcune strategie che nel corso della ricerca si sono rivelate particolarmente efficaci e funzionali, al punto da essere indicate come buone prassi, ovvero come azioni funzionali con un alto livello di condivisione generalizzabili anche in altri contesti.

\section{II rischio di esclusione sociale}

Dalle interviste raccolte emerge che le famiglie sono spesso sole, alle prese con problemi di natura linguistica ed economica, sono disorientate e vivono in condizioni di disagio e precarietà abitativa e lavorativa ${ }^{10}$. Le difficoltà di inserimento sociale e occupazionale tipiche della migrazione sono ulteriormente amplificate per le famiglie con bambini disabili che spesso non possono contare su una solida rete sociale ${ }^{11}$. Come teorizzato dallo studioso argentino Carlos Sluski, la mobilità implica per il migrante l'impoverimento del "bozzolo sociale dell'individuo", ovvero il restringimento della rete sociale in cui è inserito. Quando gli individui migrano "devono necessariamente lasciarsi alle spalle una buona parte, se non tutto, il supporto sociale di cui godono, incluso il gruppo esteso di riferimento, fatto di amici e conoscenti, il mondo tranquillizzante della famiglia allargata"12. Dal punto di vista relazionale, la migrazione si presenta dunque come un'esperienza stressante a causa della dislocazione della rete sociale e dell'elaborazione del lutto che il migrante deve affrontare a causa della perdita dei legami primari ${ }^{13}$.

II restringimento della rete sociale non garantisce un supporto di fronte ad eventi critici, come la perdita del lavoro o la disabilità di un familiare ${ }^{14}$. Dalla ricerca bolognese emerge che le famiglie migranti con minori disabili spesso non conoscono i diritti delle persone con disabilità ed entrano facilmente in conflitto con gli assistenti sociali, dinamica che spinge molti genitori al fatalismo e alla chiusura progettuale ${ }^{15}$. Diverse famiglie coinvolte nella ricerca hanno inoltre scarsi contatti con l'associazionismo su base etnica e/o culturale, spesso non conoscono l'esistenza delle associazioni dei famigliari dei disabili. L'assenza di contatti con il mondo dell'associazionismo preclude la possibilità di tessere legami significativi, utili anche per avere un aiuto concreto nella gestione del minore in orario extrascolastico ${ }^{16}$.

\footnotetext{
${ }_{10}$ ARGIROPOULOS, Dimitrios. Le famiglie, p. 143.

11 BINI, op. cit., p. 205.

12 SLUSKI, Carlos. Migrazione: la prospettiva delle reti sociali, p. 157-158.

13 Ibidem, p. 164.

${ }^{14}$ BINI, op. cit., p. 205.

15 ARGIROPOULOS, op. cit., p. 143.

${ }^{16}$ LEONARDI, Barbara. Famiglie, associazionismo e reti sociali, p. 127-129.
} 
Di fronte alle criticità sinora delineate, sarebbe opportuno intervenire sul fronte relazionale ed assistenziale per rispondere in maniera adeguata ai bisogni emergenti. Per favorire la costruzione di legami sul territorio e per garantire un sostegno alle famiglie in difficoltà, il ricercatore Argiropoulos indica alcune strategie inclusive: promuovere la formazione di gruppi cooperativi genitoriali (gruppi di migranti e gruppi misti con e senza la cittadinanza italiana); incoraggiare il coinvolgimento delle associazioni etniche e di volontariato in sinergia con la rete dei Servizi Territoriali; attivare il servizio di Assistenza Domiciliare nei casi di grave disabilità, con il coinvolgimento sia del settore pubblico che privato; introdurre a livello legislativo il rilascio del permesso di soggiorno "per motivi di sostegno alla disabilità" per favorire i ricongiungimenti familiari finalizzati alla cura e all'assistenza di parenti disabili ${ }^{17}$.

\section{Difficoltà linguistiche ed interpretative: il concetto di disabilità nelle varie culture}

Secondo la ricerca curata dalla Prof.ssa Caldin, i principali fattori che ostacolano una serena relazione tra le famiglie e i servizi sono riconducibili all'area della comunicazione: la scarsa conoscenza dell'italiano da parte dei genitori migranti e le problematiche di natura interpretativa. Si tratta di fattori interconnessi che denotano la facilità con cui il processo comunicativo rischia di sfociare in un fraintendimento ${ }^{18}$.

Di fronte alle difficoltà linguistiche dei genitori, possono essere introdotti alcuni strumenti: la traduzione nella lingua madre dei documenti riguardanti il funzionamento dei servizi e la documentazione relativa la presa in carico del minore, come il Piano Educativo Individualizzato (PEI); prevedere interventi di mediazione linguistico-culturale durante i colloqui.

Come rilevato dalla ricerca, le strategie indicate non sono però ancora consolidate e diffuse a livello territoriale; in particolare, la mediazione linguistico-culturale andrebbe valorizzata in quanto facilita la comunicazione tra due realtà diverse, non solo a livello linguistico, ma anche culturale ${ }^{19}$. Spesso infatti esistono sostanziali differenze tra i concetti di cura e di disabilità, che ricordiamo sono culturalmente condizionati, variano da cultura a cultura e, a seconda del contesto di riferimento, implicano un approccio diverso ${ }^{20}$. Se infatti il modello clinico occidentale si fonda sull'aspetto performativo del corpo e considera la malattia con oggettività e razionalità, le medicine tradizionali di alcuni Paesi si basano su una visione olistica del mondo e dell'individuo ${ }^{21}$.

\footnotetext{
17 ARGIROPOULOS, op. cit., p. 145-146.

${ }^{18}$ LEONARDI, Barbara. II punto di vista dei Servizi Territoriali, p. 154.

${ }^{19}$ FRASCÀ, Valentina. Disabilità e migrazione: problematiche e sviluppi, p. 7.

${ }^{20}$ GOUSSOT, Alain. Disabilità, rappresentazioni e mondi culturali, p. 11-13.

${ }^{21}$ TOGNETTI BORDOGNA, Mara. I colori del welfare. Servizi alla persona di fronte all'utenza che cambia, p. 11.
} 
In alcune culture la malattia è vista come un'alterazione dell'equilibrio energetico (Cina e America Latina); come la "perdita dell'anima" determinata dall'intrusione di un'entità maligna nel corpo sano (popoli gitani) oppure come una punizione divina conseguente alla violazione di un tabù ${ }^{22}$. Si tratta di rappresentazioni che influenzano il modo di reagire alla disabilità (con paura, rassegnazione o senso di colpa), il metodo di cura (medicina tradizionale del Paese di origine e/o medicina occidentale) e le aspettative sulla qualità della vita del minore disabile ${ }^{23}$. In questo contesto caratterizzato dalla tensione tra modelli culturali diversi che orientano verso la disabilità, l'utente migrante si approccia alla medicina occidentale in base alle conoscenze in suo possesso, in base ai sentimenti che nutre (fiducia/diffidenza), in base allo status sociale (permesso di soggiorno, residenza, conoscenza dell'italiano) ${ }^{24}$, a volte oscillando tra il metodo di cura occidentale e il metodo tradizionale del Paese di origine.

Nel momento in cui il migrante accede alle strutture sanitarie delle società occidentali, si possono dunque creare delle barriere in quanto il sapere scientifico e le metodologie diagnostiche usate dagli operatori non appartengono al sistema di riferimento a lui familiare ${ }^{25}$. Si tratta di un aspetto molto importante che gli operatori socio-sanitari e gli insegnanti devono conoscere per evitare che emergano conflitti ed incomprensioni. Se infatti nella maggior parte dei casi i genitori migranti accettano le cure bio-mediche tipiche del mondo occidentale, potrebbe accadere che genitori senegalesi (cultura Sérère) con un figlio disabile intellettivo si oppongano alla terapia medicoriabilitativa in quanto nella comunità di origine il comportamento "strano" del minore è considerato come il segno di vicinanza al mondo degli antenati ${ }^{26}$.

\section{Risorse inclusive per facilitare la comunicazione}

Di fronte alle difficoltà linguistiche dei genitori e alla varietà di rappresentazioni culturali sulla disabilità, come possono dialogare le conoscenze degli operatori socio-sanitari e dei docenti con il sapere dei genitori? Per promuovere il dialogo, occorre munirsi di nuovi strumenti, metodi interpretativi e strategie per interrogare il materiale etnografico di cui sono portatori i migranti ${ }^{27}$; solo con uno "sguardo antropologico attrezzato" gli operatori dell'area socio-sanitaria ed educativa possono infatti definire interventi mirati ${ }^{28}$.

${ }^{22}$ PICOZZI, Mario, SALA, Roberta, TAVANI, Mario. Riflessioni su medicina e multiculturalismo, p. 28-29.

${ }^{23}$ BINI, op. cit., p. 208.

${ }^{24}$ GOZZOLI, Caterina, REGALIA, Camillo. Migrazioni e famiglie. Percorsi, legami e interventi psicosociali, p. 217.

25 PASINI, Nicola, PICOZZI, Mario. Salute e immigrazione. Un modello teorico-pratico per le aziende sanitarie, p. 11.

${ }^{26}$ GOUSSOT, op. cit., p. 13.

${ }^{27}$ PENNAZIO, Valentina, ARMANI, Samantha, TRAVERSO, Andrea. Le famiglie migranti di bambini disabili. Progettualità e interventi educativi, p. 169.

${ }^{28}$ LEPORE, Laura. Per uno sguardo antropologico sulla disabilità: i minori disabili stranieri, p. 104. 
In primo luogo è necessario prevedere percorsi di formazione interculturale per allenare gli operatori ad assumere un atteggiamento di apertura verso I'Altro. Di fronte all'aumento di utenti migranti, gli operatori dei servizi sociosanitari e scolastici sono chiamati ad acquisire competenze e metodologie dell'area antropologica ed etnografica. Risulta auspicabile acquisire un bagaglio concettuale che metta gli operatori in grado di interpretare i riferimenti culturali dei migranti, di costruire un dialogo per portare alla luce una cultura non astratta e generale, ma radicata nel singolo, con le sue contraddizioni e ibridazioni. Attraverso questo dialogo si può costruire "una sfera comune di significati" che conduce alla nascita di un mondo simbolico condiviso ${ }^{29}$. In questi anni è inoltre emersa la consapevolezza che la formazione interculturale degli operatori è necessaria per favorire significative occasioni di scambio con le famiglie, spazi di incontro spogliati da pregiudizi e stereotipi per evitare di costruire una gerarchia tra i vari modi di pensare al fine di sostenere l'incontro tra culture diverse ${ }^{30}$.

Un'altra risorsa da valorizzare per conoscere ed interrogare il materiale etnografico dei migranti è rappresentata dalla mediazione linguistico-culturale. II mediatore linguistico-culturale non è un semplice interprete né un traduttore; la specificità del suo ruolo professionale risiede nella capacità di costruire un ponte tra il "qui" (la lingua, le persone, le istituzioni, le abitudini, la storia e le tradizioni del paese di accoglienza) e il "là" (la lingua, gli individui, la storia e le tradizioni del migrante). II mediatore mette in relazione le persone, decodifica e rende accessibile all'utente la comunicazione delle Istituzioni. Deve saper dunque intercettare gli interessi e le esigenze dell'utente decodificandoli in modo che i servizi li possano accogliere, ma deve anche chiarire all'utente il funzionamento dei servizi affinché possa utilizzarli autonomamente ${ }^{31}$. Le funzioni che sono assegnate a questa figura sono dunque notevoli: facilitare la comunicazione durante i colloqui con gli operatori; ascoltare; interpretare; tradurre; decodificare; sostenere, negoziare; orientare ${ }^{32}$. Come accennato sopra, la mediazione linguistico-culturale non è però un servizio diffuso in maniera capillare sul territorio, spesso gli interventi sono legati a progetti specifici, a risorse e a tempi limitati. Le principali criticità (contratti di lavoro spesso precari, mancanza di un riconoscimento istituzionale univoco della figura professionale, numero esiguo di corsi di formazione e di aggiornamento ad hoc) inducono molti mediatori a decidere di cambiare lavoro ${ }^{33}$. Garantire

${ }^{29}$ CAPUTO, Barbara. L'etnicizzazione della malattia in contesto migratorio. Una prospettiva antropologica, p. 124-125.

${ }^{30}$ FRASCÀ, op. cit., p. 5.

${ }^{31}$ ZANETTI, Luisa. Famiglie straniere e servizi sul territorio, p. 180.

32 JOSI, Elisabetta, PALMA, Lucia, SCIAMPLICOTTI, Fulvio. II mediatore culturale nella giustizia minorile, p. 197.

${ }^{33}$ MORANDI, Alessandro. Le buone prassi di mediazione linguistico-culturale e il ruolo di enti e associazioni nella promozione della mediazione in Toscana: esiti di un'indagine, p. 82-83 e 86-89. 
una maggiore tutela e un maggior riconoscimento professionale sono due requisiti fondamentali per evitare un ricambio continuo di figure e per costruire un servizio strutturato ${ }^{34}$. I dati della ricerca bolognese sottolineano inoltre che grazie ad interventi di mediazione linguistico-culturale la relazione tra gli operatori e le famiglie migranti risulta essere più inclusiva e serena.

II mediatore può inoltre rappresentare una valida risorsa per chiarire alla famiglia come funziona la presa in carico del minore disabile, specificando il ruolo dei servizi socio-sanitari (area medico-riabilitativa) e il ruolo della scuola (area educativa) nel percorso di integrazione. Per semplificare la complessità degli interventi gestiti dall'équipe medico-riabilitativa spesso "numerosa" (questo aspetto dipende dalla gravità del deficit di ciascun bambino; in genere un'équipe è composta da: neuropsichiatra infantile, logopedista, psicomotricista, educatore, assistente alla comunicazione, docente di sostegno e insegnante curricolare), sarebbe auspicabile introdurre capillarmente anche un'altra risorsa: la figura del case manager.

Il case manager è il professionista che nel team di lavoro, oltre a svolgere il proprio ruolo (ad esempio, logopedista o neuropsichiatra), in accordo con le altre figure del gruppo di esperti, ricopre la funzione di interlocutore diretto con la famiglia e fornisce periodicamente informazioni sul progetto didattico e riabilitativo del minore ${ }^{35}$.

All'interno del lavoro di rete che coinvolge gli Enti preposti alla presa in carico del minore disabile, la formazione interculturale rivolta ad operatori ed insegnanti, la mediazione linguistico-culturale e la figura del case manager sono risorse che generano un nuovo orientamento metodologico, una pratica meticcia che struttura la fase di accoglienza, di accompagnamento e di cura del minore con disabilità e della sua famiglia. Si tratta di un approccio multidisciplinare che permette di analizzare il fenomeno della migrazione e della disabilità da più prospettive. La pratica meticcia in campo riabilitativo e pedagogico si basa sul dialogo tra diversi punti di vista, tra diversi modelli culturali e tra diverse discipline (come etnografia, antropologia culturale, Pedagogia Speciale, Pedagogia Interculturale, etnopsichiatria) ${ }^{36}$.

\section{Conclusioni}

Il quadro delineato dalla ricerca svolta a Bologna evidenzia che attraverso la conoscenza delle condizioni delle famiglie e la mappatura dei bisogni emergenti, le Istituzioni chiamate a collaborare nella presa in carico del minore

\footnotetext{
${ }^{34}$ ALBERTINI, Valentina. Gli ambiti della mediazione linguistico-culturale: aspetti positivi e criticità emergenti, p. 56.

${ }^{35}$ CALDIN, ARGIROPOULOS, DAINESE, op. cit., p. 16.

${ }^{36}$ GOUSSOT, Alain. Bambini stranieri con bisogni speciali: rappresentazione della disabilità dei figli da parte delle famiglie migranti e degli insegnanti, p. 22.
} 
disabile e della sua famiglia possono adottare strumenti e risorse in grado di definire scenari inclusivi.

Di fronte a difficoltà di natura linguistica ed interpretativa, risulta necessario rinnovare le modalità comunicative (traduzione dei documenti, presenza del mediatore linguistico-culturale durante i colloqui), introdurre strategie per facilitare la relazione con la famiglia (formazione interculturale degli operatori, nominare il case manager nel gruppo di esperti, fissare incontri periodici), favorire pratiche interdisciplinari (promuovere il dialogo tra diversi modelli culturali e varie discipline) per generare un orientamento metodologico meticcio. Solo un'impostazione globale, capace di affrontare il fenomeno da più prospettive, può infatti restituire un quadro approfondito della situazione e sollecitare risposte adeguate ai bisogni dei minori e delle loro famiglie ${ }^{37}$.

Di fronte alla mancanza di protocolli e linee guida ministeriali per definire strategie di accoglienza e di accompagnamento, ad oggi la ricerca "Alunni con disabilità, figli di migranti" costituisce un prezioso quadro di riferimento dal quale attingere buone pratiche e utili indicazioni sul tema della disabilità in contesti migratori.

\section{Bibliografia}

ALBERTINI, Valentina. Gli ambiti della mediazione linguistico-culturale: aspetti positivi e criticità emergenti. In ALBERTINI, Valentina; CAPITANI, Giulia. La mediazione linguistico-culturale. Stato dell'arte e potenzialità. Pisa: Quaderni del Centro Servizi Volontariato Toscana, n. 47, 2010, p. 39-68.

ARGIROPOULOS, Dimitrios. Le famiglie. In CALDIN, Roberta (a cura di). Alunni con disabilità, figli di migranti. Approcci culturali, questioni educative, prospettive inclusive. Napoli: Liguori Editore, 2012, p. 135-146.

BALSAMO, Franca. Famiglie di migranti. Trasformazioni dei ruoli e mediazione culturale. Roma: Carocci Editore, 2003.

$\mathrm{BINI}$, Emanuela. Famiglie migranti con minori disabili. Prospettive inclusive nel sistema socio-sanitario e scolastico italiano. Culture e Studi del sociale - CuSSoc, v. 2, n. 2, Dicembre 2017, p. 203-211. Disponibile su: <http://www.unisa.it/ uploads/14620/201702.pdf>. Visitato il 03.01.2018.

BINI, Emanuela. Disabilità e migrazione: pratiche di accoglienza nei servizi sociosanitari e strategie inclusive a scuola. In PROFANTER, Annemarie (a cura di). Kulturen im Dialog IV/Culture in Dialogo IV/Cultures in dialogue IV. Francoforte: Peter Lang Edition, 2016, p. 207-216.

BLANGIARDO, Giancarlo; TERZERA, Laura. Le famiglie immigrate: percorsi e progetti di un universo in continua evoluzione. In SCABINI, Eugenia; ROSSI, Giovanna (a cura di). La migrazione come evento familiare. Milano: Vita e Pensiero, 2008.

CALDIN, Roberta; ARGIROPOULOS, Dimitrios; DAINESE, Roberto. Genitori

37 BINI, op. cit., p. 209. 
migranti e figli con disabilità. Le rappresentazioni dei professionisti e le percezioni delle famiglie. Ricerche di Pedagogia e Didattica, v. 5, n. 1, 2010. Disponibile su: $<$ https://rpd.unibo.it/article/view/1740>. Visitato il 25.01.2018.

CALDIN, Roberta (a cura di). Alunni con disabilità, figli di migranti. Approcci culturali, questioni educative, prospettive inclusive. Napoli: Liguori Editore, 2012.

CALDIN, Roberta. Alunni con disabilità figli di migranti. Intrecci, contrasti, prospettive. Educazione Interculturale. Culture, esperienze, progetti, v. 9, n. 1, 2011, p. 27-37.

CAPUTO, Barbara. L'etnicizzazione della malattia in contesto migratorio. Una prospettiva antropologica. In PASINI, Nicola; PICOZZI, Mario. Salute e immigrazione. Un modello teorico-pratico per le aziende sanitarie. Milano: Franco Angeli, 2005, p. 99-132.

CODINI, Ennio. Gli aspetti normativi. In FONDAZIONE ISMU. Ventiduesimo Rapporto sulle migrazioni 2016. Milano: Franco Angeli, 2016, p. 103-110.

CORNEO, Daniela. Alunni stranieri tra i banchi: Bologna supera Milano e Roma. II rapporto Ismu: qui il maggior numero di classi dove si sfora il tetto del $30 \%$. 09/12/2017. Disponibile su: <http://corrieredibologna.corriere.it/bologna/cron aca/17_dicembre_09/alunni-stranieri-banchi-bologna-supera-milano-roma-

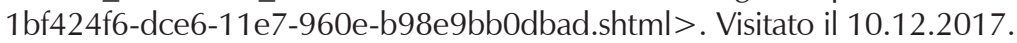

DAINESE, Roberto; GORI, Elisa. Istituzioni educative e integrazione scolastica: risorse e criticità. In CALDIN, Roberta (a cura di). Alunni con disabilità, figli di migranti. Approcci culturali, questioni educative, prospettive inclusive. Napoli: Liguori Editore, 2012, p. 79-126.

FONDAZIONE Ismu; MIUR. Rapporto nazionale sugli alunni con cittadinanza non italiana. La scuola multiculturale nei contesti locali. 1/2016. Disponibile su: <http://www.condicio.it/allegati/219/AlunniCittadinanzaNonltaliana_ISMU_ MIUR_2014_2015.pdf > . Visitato il 26.11.2017.

FRASCÀ, Valentina. Disabilità e migrazione: problematiche e sviluppi. Quaderni di Intercultura, Anno III, 2011, p. 1-9.

GOUSSOT, Alain. Disabilità, rappresentazioni e mondi culturali. Educazione interculturale. Culture, esperienze, progetti, v. 9, n. 1, 2011, p. 11-26.

GOUSSOT, Alain. Bambini stranieri con bisogni speciali: rappresentazione della disabilità dei figli da parte delle famiglie migranti e degli insegnanti. Ricerche di Pedagogia e Didattica, v. 5, n. 1, 2010. Disponibile su: <https://rpd.unibo.it/ article/view/1763/1140>. Visitato il 25.01.2018.

GOUSSOT, Alain. Identità meticce, pratiche meticce. Suggestioni metodologiche nell'ambito della cura, riabilitazione, educazione. Animazione sociale, n. 210, 2007.

GOZZOLI, Caterina; REGALIA, Camillo. Migrazioni e famiglie. Percorsi, legami e interventi psicosociali. Bologna: Il Mulino, 2005.

JOSI, Elisabetta; PALMA, Lucia; SCIAMPLICOTTI, Fulvio. II mediatore culturale nella giustizia minorile. In ANDOLFI, Maurizio (a cura di). La mediazione culturale. Tra I'estraneo e il familiare. Milano: Franco Angeli, 2003, p. 194-211.

LEONARDI, Barbara. Famiglie, associazionismo e reti sociali. In CALDIN, Roberta (a cura di). Alunni con disabilità, figli di migranti. Approcci culturali, questioni educative, prospettive inclusive. Napoli: Liguori Editore, 2012, p. 127-134. 
LEONARDI, Barbara. II punto dei vista dei Servizi Territoriali. In CALDIN, Roberta (a cura di). Alunni con disabilità, figli di migranti. Approcci culturali, questioni educative, prospettive inclusive. Napoli: Liguori Editore, 2012, p. 147-160.

LEPORE, Laura. Per uno sguardo antropologico sulla disabilità: i minori disabili stranieri. Minori giustizia. Rivista interdisciplinare di studi giuridici, psicologici, pedagogici e sociali sulla relazione tra minorenni e giustizia, n. 3, 2010. Milano: Franco Angeli, p. 94-105.

MORANDI, Alessandro. Le buone prassi di mediazione linguistico-culturale e il ruolo di enti e associazioni nella promozione della mediazione in Toscana: esiti di un'indagine. In ALBERTINI, Valentina; CAPITANI, Giulia. La mediazione linguistico-culturale. Stato dell'arte e potenzialità. Pisa: Quaderni del Centro Servizi Volontariato Toscana, n. 47, 2010, p. 71-104.

OSSERVATORIO REGIONALE SUL FENOMENO MIGRATORIO. Rapporto "Cittadini stranieri in Emilia Romagna. Residenti e dinamiche demografiche", 2017. Disponibile su: <http://sociale.regione.emilia-romagna.it/immigrati-estranieri/temi/archivio-dati/focus-2 > . Visitato il 30.11.2017.

PASINI, Nicola; PICOZZI, Mario. Salute e immigrazione. Un modello teorico-pratico per le aziende sanitarie. Milano: Franco Angeli, 2005.

PENNAZIO, Valentina; ARMANI, Samantha; TRAVERSO, Andrea. Le famiglie migranti di bambini disabili. Progettualità e interventi educativi. Rivista Italiana di Educazione Familiare, n. 1, 2015, p. 167-182.

PICOZZI, Mario; SALA, Roberta; TAVANI, Mario. Riflessioni su medicina e multiculturalismo. In PASINI, Nicola; PICOZZI, Mario. Salute e immigrazione. Un modello teorico-pratico per le aziende sanitarie. Milano: Franco Angeli, 2005, p. 23-42.

SLUSKI, Carlos. Migrazione: la prospettiva delle reti sociali. In SCABINI, Eugenia; ROSSI, Giovanna (a cura di). La migrazione come evento familiare. Milano: Vita e pensiero, 2008.

TOGNETTI BORDOGNA, Mara (a cura di). I colori del welfare. Servizi alla persona di fronte all'utenza che cambia. Milano: Franco Angeli, 2004.

ZANETTI, Luisa. Famiglie straniere e servizi sul territorio. In MARAZZI, Antonio (a cura di). Voci di famiglie immigrate. Milano: Franco Angeli, 2005, p. 172-192.

Articolo ricevuto il 31.01.2018

Accettato per la pubblicazione il 19.02.2018

Received for publication in January 315t, 2018

Accepted for publication in February 19 ${ }^{\text {th }}, 2018$

ISSN impresso 1980-8585

ISSN eletrônico 2237-9843

http://dx.doi.org/10.1590/1980-85852503880005212 